\title{
BOSON AND DIBOSON PRODUCTION AT ATLAS
}

\author{
Manuela Venturi ${ }^{a}$ \\ On behalf of the ATLAS Collaboration \\ Fakultät für Mathematik und Physik, Albert-Ludwigs-Universität, \\ Freiburg im Breisgau, Germany \\ Abstract. Results obtained with the ATLAS detector [1] at the LHC, with $7 \mathrm{TeV}$ \\ pp-collisions and an integrated luminosity up to $1 \mathrm{fb}^{-1}$, are presented for many \\ electroweak processes. In particular, $W$ and $Z$ cross section measurements are \\ discussed, along with $W$ charge asymmetry, $W+$ jets production, $W W, Z Z$ and \\ $W Z$ cross sections and their gauge couplings.
}

\section{Introduction}

In the LHC era, it is crucial to gain a good understanding of the electroweak (EWK) sector of the Standard Model (SM), both in its own right and as a prerequisite to discovery searches. In the following, the most up-to-date measurements of $W, Z, W+$ jets, $W W, Z Z$ and $W Z$ cross sections will be reviewed, along with that of the $W$ charge asymmetry. There are both theorethical and experimental motivations for studying these processes.

From an experimental point of view, $W$ and $Z$ leptonic decays are used to benchmark the detector, i.e. to establish efficiencies of lepton triggers and reconstruction, energy and angular resolution, energy scales, etc.

On the other hand, from a theorethical perspective, these processes represent a stringent test of perturbative QCD (pQCD) at the TeV scale, allowing to compare the measured quantities with their predictions at next-to-leadingorder (NLO) or next-to-next-to-leading-order (NNLO). Some of the EWK measurements, namely $W$ charge asymmetry and $Z$ rapidity spectrum, allow the determination of parton distribution functions (PDFs) in a new energy regime, and are being used already to diminish PDF uncertainties. Moreover, diboson cross sections are expected to be sensitive to physics beyond the SM. Lastly, searches like that for the Higgs boson and for Supersymmetry require a precise knowledge of the EWK SM sector, since it represents an important background.

\section{Boson Physics}

\section{$2.1 W$ and $Z$ cross section measurements}

The analysis [2] uses data collected by ATLAS during 2010, corresponding to around $35 \mathrm{pb}^{-1}$ of integrated luminosity, to determine the cross sections times leptonic branching ratios, $\sigma_{W^{ \pm}} \cdot \operatorname{BR}(W \rightarrow \ell \nu)$ and $\sigma_{Z / \gamma^{*}} \cdot \operatorname{BR}\left(Z / \gamma^{*} \rightarrow\right.$ $\ell \ell)$, where $\ell=e, \mu$. The integrated and differential cross sections are measured in the fiducial volume of the ATLAS detector, using the equation: $\sigma_{\text {fid }}=$

\footnotetext{
${ }^{a}$ e-mail: manuela.venturi@cern.ch
} 
$(N-B) /\left(C_{W, Z} \cdot L\right)$, where $N$ is the number of candidate events observed in data, $B$ the number of background events and $L$ the integrated luminosity corresponding to the run selections and trigger employed. The efficiency factor $C_{W, Z}$ determines the fiducial region of the detector, defined as a function of the transverse momentum, $p_{T}$, and pseudorapidity, $\eta$, of the final state leptons.

Muons are required to be combined, i.e. reconstructed using both the Inner Detector (ID) and the Muon Spectrometer (MS). They are requested to satisfy quality cuts, have $p_{T}>20 \mathrm{GeV}$ and $|\eta|<2.4$.

Electrons are reconstructed from their ID tracks and calorimeter informations, with quality cuts applied on both. An electron candidate has $p_{T}>20$ $\mathrm{GeV}$ and $|\eta|<2.47$, excluding the region $1.37<|\eta|<1.52$, where the transition between the barrel and the end-cap calorimeters is. For $Z \rightarrow e e$, the range up to $\eta=4.9$ is added to the analysis, allowing one of the electrons to be in the forward region.

Additional selection criteria are applied to reduce the background and define the signal regions. For $W \rightarrow \ell \nu$, transverse missing energy and mass have to be respectively $E_{T}^{\text {miss }}>25 \mathrm{GeV}$ and $m_{T}>40 \mathrm{GeV}$, where $m_{T}=$ $\sqrt{2 p_{T}^{\ell} E_{T}^{\text {miss }}\left(1-\cos \left(\phi^{l}-\phi^{\nu}\right)\right)}$. For $Z \rightarrow \ell \ell$, leptons are required to have same flavour and opposite charge, while their invariant mass has to be $66<m_{\ell \ell}<$ $116 \mathrm{GeV}$.

In all analyses, QCD multijet background is determined from data, with methods varying according to the analysis (template fit to the $E_{T}^{\text {miss }}$ distribution for $W \rightarrow e \nu$, control regions for the others). EWK and $t \bar{t}$ backgrounds are negligible and are constrained via $\mathrm{MC}$ simulations.

Figure 1 [2] shows the measured $W^{+}, W^{-}, W^{ \pm}$and $Z$ cross sections in the fiducial regions, compared with theorethical predictions using different PDF sets. The agreement is good in all cases.

\section{2 $W$ charge asymmetry}

The same selection shown in (2.1) is used to determine the charge asymmetry $A$ for the lepton from the $W$ decay, in bins of pseudorapidity, defined as: $A=\left(d \sigma_{W_{\ell^{+}}} / d \eta_{\ell}-d \sigma_{W_{\ell^{-}}} / d \eta_{\ell}\right) /\left(d \sigma_{W_{\ell^{+}}} / d \eta_{\ell}+d \sigma_{W_{\ell^{-}}} / d \eta_{\ell}\right)$. This is a powerful measurement to probe the inner structure of the proton since many systematic uncertainties cancel in the ratio, while the dependence on $\eta_{\ell}$ allows to explore different regions of $x$. Results are shown in Figure 2 (left) [2], with a comparison to NNLO calculations.

\section{$2.3 W+$ jets}

Another measurement performed with the selection described in (2.1), is the production cross section for a $W$ boson in association with jets [3]. Jets are reconstructed using an anti- $k_{T}$ algorithm with a radius parameter $R=0.4$. 

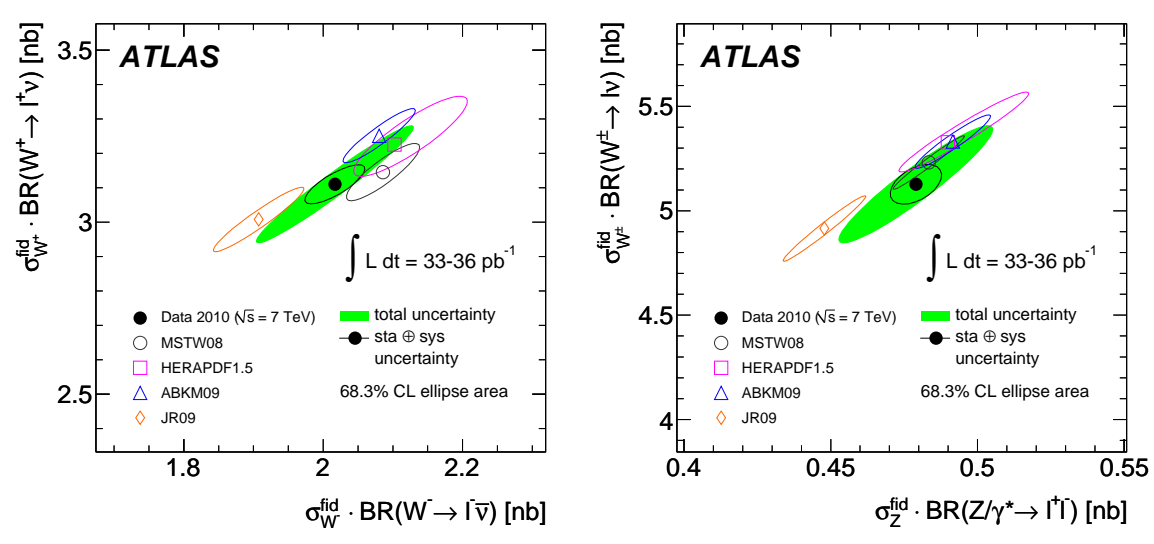

Figure 1: Left: $W^{+}$vs $W^{-}$fiducial cross section; right: $W^{ \pm}$vs $Z$ fiducial cross section. In both cases, a comparison with predictions from four different PDF sets is shown.

These jets are required to have $E_{T}>20 \mathrm{GeV}$, rapidity $|y|<2.8$ and not to be close $(\Delta R>0.5)$ to any lepton candidate. Figure 2 (right) shows the cross section ratio as a function of jet multiplicity for the muon channel. $\mathrm{MC}$ predictions agree with data at NLO, but not at LO (Pythia), as expected.

\section{Diboson Physics}

\subsection{WW cross section measurement}

The measurement of the $W W$ production cross section at the LHC provides an important test of the SM through its sensitivity to triple gauge boson couplings (TGCs), which result from the non-Abelian structure of the gauge symmetry group, $\mathrm{SU}(2)_{L} \times \mathrm{U}(1)_{Y}$. Furthermore, non-resonant $W W$ production is an irreducible background in searches for the Higgs boson in the same final state.

The analysis [4] uses an integrated luminosity of $1.02 \mathrm{fb}^{-1} ; W W$ events are reconstructed using leptonic $(\ell=e, \mu)$ decays of the $\mathrm{W}$ boson. The resulting final state has two high- $p_{T}$ charged leptons and substantial $E_{T}^{\text {miss }}$. The major backgrounds are Drell-Yan, top quark, $W+$ jet and diboson $(W Z, Z Z$ and $W \gamma)$ production.

Muons and electrons are defined as in the standard $W$ analysis, described in (2.1), apart from a more stringent cut on the $p_{T}$ of the leading electron, $p_{T}>25 \mathrm{GeV}$. Jets, reconstructed using an anti- $k_{T}$ algorithm as in (2.3), are required to have $E_{T}>30 \mathrm{GeV}$ and $|y|<4.5$.

The selection is divided into three channels: $e e, \mu \mu$ and $e \mu$. After requiring two leptonic candidates with opposite charge, originating from the primary 

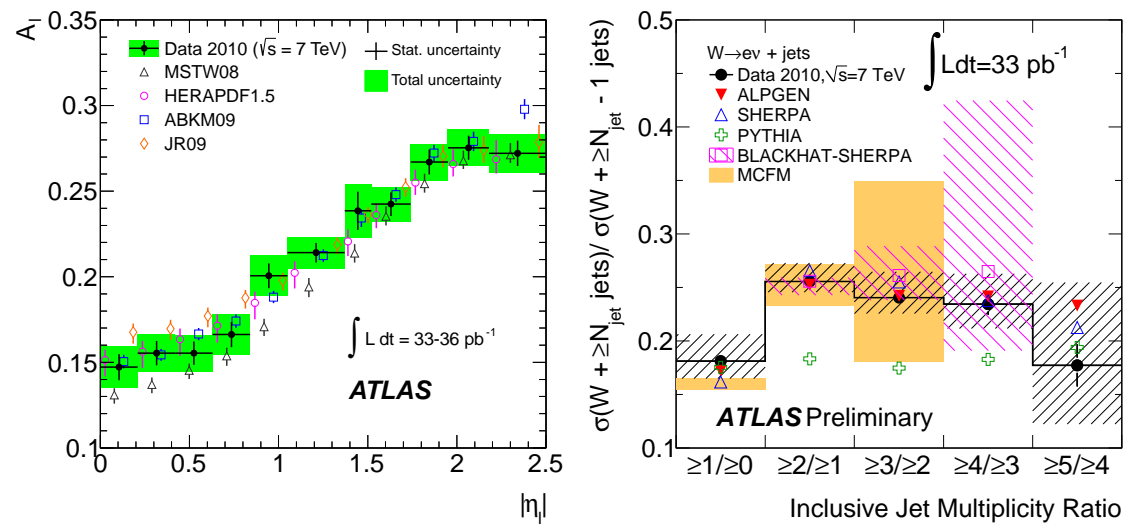

Figure 2: Left: Charge asymmetry in $W$ decays as a function of $\eta_{\ell}$, compared with NNLO predictions; right: cross section ratio as a function of jet multiplicity in the muon channel.

vertex of the event, cuts are applied on the dilepton invariant mass $\left(m_{l l}>10\right.$ $\mathrm{GeV}$ for $e \mu, m_{l l}>15 \mathrm{GeV}$ and $\left|m_{l l}-m_{Z}\right|>15 \mathrm{GeV}$ for $e e$ and $\left.\mu \mu\right)$ and on the relative missing transverse energy $\left(E_{T, \text { Rel }}^{\text {miss }}>25,40,45\right.$ for $e \mu, e e, \mu \mu$ respectively). Jets are fully vetoed, in order to reduce the $t \bar{t}$ background.

The total cross section is extrapolated from the fiducial region, with a combination of the three channels, yielding $48.2 \pm 4.0$ (stat) \pm 6.4 (syst) \pm 1.8 (lumi) $\mathrm{pb}$, which agrees nicely with the theoretical NLO prediction of $46 \pm 3 \mathrm{pb}$.

\section{2 $Z Z$ and $W Z$ cross section measurements}

In the SM, ZZ production proceeds predominantly via the t-channel, while the s-channel vertices $(Z Z Z, Z Z \gamma)$ - also known as neutral Trilinear Gauge Couplings (nTGCs) - are forbidden. Non-zero nTGCs would lead to an enhancement of the $Z Z$ cross section, and their observation would be an hint of physics beyond the SM. We also note that this process is an irreducible background for the search $H \rightarrow Z Z$, and thus crucial to be constrained.

Three final states are considered in the analysis $(e e e e, \mu \mu \mu \mu, e e \mu \mu)$, which also employs $1.02 \mathrm{fb}^{-1}[5]$. Leptons are defined closely to the $W W$ selection, as described in (3.1); they are then associated in pairs of same flavour and opposite charge to form $Z$ candidates, with $\left|m_{\ell \ell}-m_{Z}\right|<15 \mathrm{GeV}$.

The cross section resulting from the combination of all the channels, for a total of $12 Z Z$ candidates, is found to be $8.4_{-2.3}^{+2.7}$ (stat) ${ }_{-0.7}^{+0.4}$ (syst) \pm 0.3 (lumi) $\mathrm{pb}$, which is statistically consistent with the SM NLO expectation of $6.5_{-0.2}^{+0.3}$ pb. The corresponding exclusion limits for nTGCs $\neq 0$ are shown in Figure 3 (left), and are found to be already competitive with those obtained from CDF 
and LEP, after a much longer data taking period.

With a similar selection, the $W Z$ cross section is also measured, yielding $21.1_{-2.8}^{+3.1}$ (stat) \pm 1.2 (syst) pb, compatible with the SM NLO expectation of $17.2_{-0.8}^{+1.2} \mathrm{pb}$. Limits on the anomalus gauge couplings (aTGCs) are also derived, no hint of physics beyond the SM is found (Figure 3 (right)).
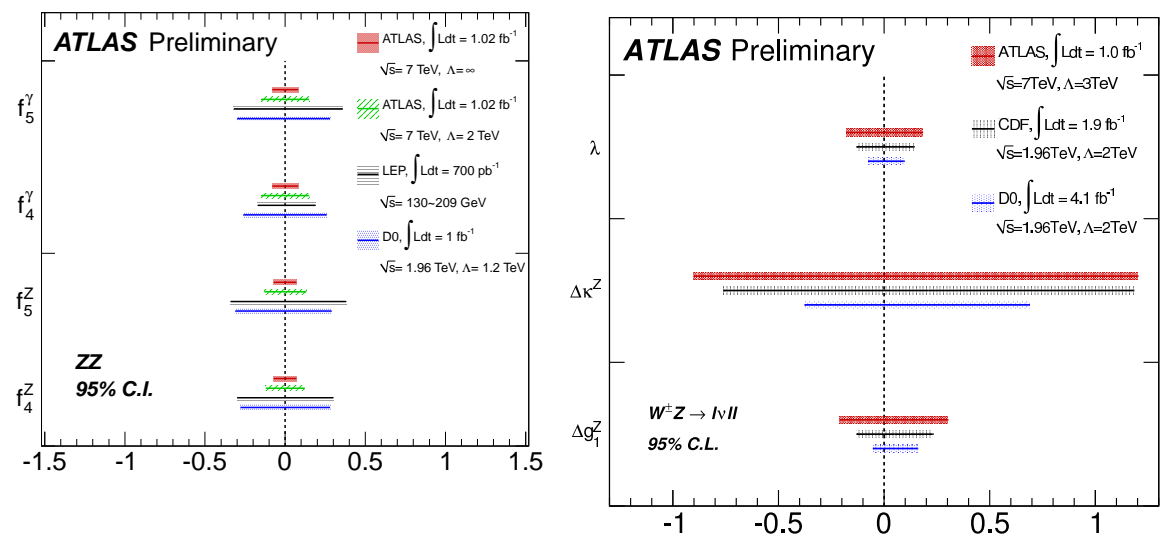

Figure 3: Left: Limits on nTGCs; right: limits on aTGCs. In both cases, ATLAS results are compared with those from other collider experiments.

\section{Conclusions}

Measurements performed by the ATLAS experiment with collision data collected in 2010 and in the first half of 2011 have been shown, namely the cross sections for $W, Z, W+$ jets, $W W, Z Z$ and $W Z$ leptonic decays, along with the $W$ charge asymmetry. Others - such as $Z$ rapidity spectrum, $Z+$ jets, $W \gamma, Z \gamma$ - could not be discussed here for brevity's sake. All results are found to be in nice agreement with the SM NLO expectation, with no deviation from the SM observed.

\section{References}

[1] The ATLAS Collaboration, JINST 3 (2008) S08003.

[2] The ATLAS Collaboration, arXiv:1109.5141 [hep-ex].

[3] The ATLAS Collaboration, arXiv:1109.5141 [hep-ex].

[4] The ATLAS Collaboration, ATLAS-CONF-2011-110

[5] The ATLAS Collaboration, ATLAS-CONF-2011-107.

[6] The ATLAS Collaboration, ATLAS-CONF-2011-099. 